Draft VERSion OCTOBER 22, 2018

Preprint typeset using $\mathrm{L}_{\mathrm{T}} \mathrm{EX}$ style emulateapj v. 08/22/09

\title{
ALIGNMENT OF DUST WITH MAGNETIC INCLUSIONS: RADIATIVE TORQUES AND SUPERPARAMAGNETIC BARNETT AND NUCLEAR RELAXATION
}

\author{
A. LAzArian \& Thiem HoAng \\ Dept. of Astronomy, University of Wisconsin, Madison, WI53706; lazarian; hoang@astro.wisc.edu \\ Draft version October 22, 2018
}

\begin{abstract}
We consider grains with superparamagnetic inclusions and report two new condensed matter effects that can enhance the internal relaxation of the energy of a wobbling grain, namely, superparamagnetic Barnett relaxation, as well as, an increase of frequencies for which nuclear relaxation becomes important. This findings extends the range of grain sizes for which grains are thermally trapped, i.e. rotate thermally, in spite of the presence of uncompensated pinwheel torques. In addition, we show that the alignment of dust grains by radiative torques gets modified for superparamagnetic grains, with grains obtaining perfect alignment with respect to magnetic fields as soon as the grain gaseous randomization time gets larger than that of paramagnetic relaxation. The same conclusion is valid for the mechanical alignment of helical grains. If observations confirm that the degrees of alignment are higher than radiative torques can produce alone, this may be a proof of the presence of superparamagentic inclusions.

Subject headings: polarization -dust extinction -ISM: magnetic fields
\end{abstract}

\section{INTRODUCTION}

Alignment of dust in magnetic fields is one of the astrophysical problems of the longest standing. Discovered by Hiltner (1949) and Hall (1949) via observation of the polarization of starlight attenuated by interstellar dust, aligned grains are shown to trace magnetic fields in various environments, from interplanetary space (see Rosenbush et al. 2007) to circumstellar regions (see Aitken et al. 2002; Cho \& Lazarian 2005).

The presently-accepted dominant alignment process is based on the action of radiative torques (RATs) on irregular grains (see Lazarian 2007 for a review). This process was proposed by Dolginov \& Mytrophanov (1976) and extended, corrected and elaborated in the subsequent publications (Lazarian 1995; Draine 1996; Draine \& Weingartner 1996, 1997, henceforth, DW96 and DW97; Weingartner \& Draine 2003, Weingartner 2006; Lazarain \& Hoang 2007a, henceforth LH07; Hoang \& Lazarian 2008). This has induced theory-based modeling of polarized radiation from molecular clouds (Cho \& Lazarian 2005; Pelkonen et al. 2007, Bethell et al. 2007) and accretion disks (Cho \& Lazarian 2007).

While DW96 professed the omnipotence of RATs for aligning interstellar grains, the physics involved happens to be more complex than it was initially anticipated. In particular, an analytical model (AMO) for RATs proposed in LH07 shows that, for a variety of the directions between the magnetic field and the radiation flux, the alignment is not perfect (see also a particular example of this in Weingartner \& Draine 2003 and more details in Hoang \& Lazarian 2008). This causes the justified worries whether all the essential physical processes has been accounted for to explain the high degrees of interstellar polarization (see Whittet et al. 2008).

In LH07a we showed that ordinary paramagnetic relaxation does not affect the RAT alignment in most astrophysical environments, e.g., in interstellar gas. This does not preclude inclusions with strong magnetic re- sponse, e.g. superparamagnetic inclusions, to affect the RAT alignment. Such grains were discussed in Jones \& Spitzer (1967) and also Martin (1995). Given the abundance of iron in dust, this would not be a far fetched suggestion. Moreover studies of interstellar grains caught in the Earth atmosphere (see Bradley 1994; Goodman \& Whittet 1995), as well as, laboratory studies of the silicate films (see Djouadi et al. 2007) are suggestive of the abundance of such inclusions. Below in $\S 2$ we consider both the effect of superparamagnetic inclusions on grain internal relaxation, i.e. on the relaxation that may induce grain to rotate about its axes of maximal inertia (Purcell 1979; Lazarian 1994; Lazarian \& Roberge 1997; Lazarian \& Efroimsky 1998; Lazarian \& Draine 1999ab, henceforth LD99ab) and on the RAT alignment (in §3).

\section{BARNETT AND NUCLEAR RELAXATION IN SUPERPARAMAGNETIC GRAINS}

A general susceptibility of the grain with superparamagnetic inclusions is given in Draine \& Lazarian (1999). If, however, we consider only low frequency response of the material, superparamagnetic inclusions containing $N_{c l}$ atoms act in the external magnetic field as magnetic moments of the moment $N_{c l} \mu$, where $\mu$ is the moment of an individual atom. This increases the grain magnetic susceptibility by a factor of $N_{c l}$, which may vary from approximately 10 to $10^{6}$ (see Kneller \& Laborsky 1963; Billas et al. 1994). The enhanced susceptibility affects the internal relaxation through the Barnett effect.

Barnett effect (Barnett 1915) can be well understood classically (see LD99a for a quantum mechanical treatment). If one considers, instead of an electron spin, a gyroscope with a momentum J. As the grain rotates with angular velocity $\boldsymbol{\Omega}$, the torque acting on a spin is $\frac{d}{d t} \mathbf{J}=\boldsymbol{\Omega} \times \mathbf{J}$. The equivalent torque can be induced by a magnetic field $\mathbf{H}_{B}$ acting along the grain angular velocity axis, i.e. $1 / c \mu \times \mathbf{H}_{B}$, which provides the Barnettequivalent field $H_{e q v}=\frac{J c}{\mu} \Omega$. The magneto-mechanical ratio for an electron is $\gamma=\mu /(J c)=e / m c$, where $e$ and 
$m$ are the electron charge and mass, respectively. Thus the Barnett-equivalent field is $H_{e q v}=\Omega / \gamma$.

The Barnett-equivalent magnetic field induces grain magnetization $\mathbf{M}=\chi \mathbf{H}_{e q v}=\chi \boldsymbol{\Omega} / \gamma$. Purcell (1979) noticed that when a grain wobbles, the precession of $\boldsymbol{\Omega}$ in grain axes induces the precession of the vector of magnetization M, which causes energy dissipation. For a grain at an absolute zero temperature this would result in the grain getting to rotate about its axis corresponding to the maximal moment of inertia. For grains at finite temperatures, the relaxation induces important coupling between rotational and vibrational degrees of freedom, which results in many important effects (Lazarian 1994, Lazarian \& Roberge 1997, LD99a,b). We expect for grains with enhanced magnetic susceptibilities, i.e. larger $\chi$ both the magnetization and the relaxation that this magnetization entails to be enhanced.

To quantify the effect, consider the internal relaxation associated with the Barnett magnetization of a wobbling oblate grain. The angle $\theta$ between the axis of major inertia $\mathbf{a}_{1}$ and the angular momentum $\mathbf{J}$ changes according to LD99a as

$$
\frac{d \theta}{d t}=-(h-1) \frac{V J^{2}}{I_{\|} I_{\perp}^{2} \gamma^{2}}(\chi " / \omega) \sin \theta \cos \theta f(h, \theta),
$$

where $I_{\|}$and $I_{\perp}$ are the moments of inertial parallel and perpendicular to the axis of major inertia, $h \equiv I_{\|} / I_{\perp}$, $\omega=(h-1) J \cos \theta / I_{\|}$is the precession frequency, $V$ is the grain volume, $\chi$ " is the imaginary part of magnetic susceptibility, and $f(h, \theta)$ is a function of order unity that takes into account details of spin-lattice coupling. For sufficiently slow rotation, e.g. $\Omega<10^{6}$ this function is identically unity and Eq. (11) coincides with the result in Purcell (1979).

If the magnetic moment of electrons on the outer partially-filled shell is $p \mu_{B}$, where $\mu_{B} \equiv e \hbar / 2 m_{e} c$ is the Bohr magneton, while for bulk Fe we take $p=2.22$ (Morish 1980), then for zero-frequency susceptibility of a grain is (see Draine 1996)

$$
\chi_{\text {sup }}(0) \approx 1.2 \times 10^{-2} N_{c l} f_{p} \hat{n}_{23} \hat{p}_{3}^{2} \hat{T}_{15}^{-1},
$$

where $f_{p} \approx 0.01$ is the fraction of Fe atoms, $\hat{n}_{23} \equiv$ $/\left(10^{23} \mathrm{~cm}^{-3}\right)$ and $\hat{T}_{15} \equiv T /(15 \mathrm{~K})$ are normalized grain density and temperature, respectfully. Superparamagnetic clusters undergo thermally activated remagnetization at a rate

$$
\tau_{s p}^{-1} \approx \nu_{0} \exp \left[-N_{c l} T_{a c t} / T\right]
$$

where $\nu_{0} \approx 10^{9}$ and $T_{a c t} \approx 0.011 K$ (see Morish 1980$)$

Adopting critically damped susceptibility from Draine \& Lazarian (1999) one can write the imaginary part of the grain susceptibility as $\chi "=\chi_{\text {sup }}(0) \omega \tau /\left(1+(\omega \tau / 2)^{2}\right)^{2}$ . Combining the latter expression for $\chi$ " with Eqs. (1) and (2) one obtains for an $a \times a \sqrt{3} \times a \sqrt{3}$ brick the time of alignment equal to

$$
\tau_{\text {Bar }, \text { sup }} \approx 10^{8} \hat{\rho}^{2} a_{-5}^{7} \frac{1}{N_{c l}}\left(\frac{J_{d}}{J}\right)^{2} \times\left(1+\left(\frac{\omega_{1} \tau}{2}\right)^{2}\right)^{2},
$$

which is a factor $\sim N_{c l}$ smaller than the usual Barnett relaxation time.
The competing process of internal relaxation, namely, "nuclear relaxation" was introduced in LD99b. While the magnetization arising from nuclear spins of a rotating grain is $\sim 1000$ times smaller than the magnetization arising from electron spins, the nuclear relaxation $\sim H_{\text {eqv }}^{2}$ is approximately $\sim 10^{6}$ times faster than the Barnett relaxation in normal paramagnetic material. This difference in the value of Barnett-equivalent field $H_{e q v}$ can be understood if we recall that the flipping of both electron and nuclear spins in order to get spins mostly parallel to grain angular velocity is entirely mechanical effect. For a given temperature the equilibrium excess of oriented spins depends only the angular momentum of the spin and the temperature (see Lazarian \& Draine 1999a). For both nuclear and electron spins the former values are equal of $\hbar$. However, to get the same excess of orientation acting by external magnetic field on much weaker nuclear moments, one has to use a stronger magnetic field.

Superparamagnetism does not change the amplitude of the nuclear relaxation rate, but, nevertheless, affects the rate of the wobbling at which the relaxation gets saturated. Indeed, the relaxation of the system of spins depends on the rate of spin precession in the internal varying magnetic field of the neighboring magnetic moments. In the medium with magnetic moments $\mu_{m}$ with density $n_{m}$ one can use van Vleck (1937) expression for the internal magnetic field $H_{i} \approx 3.8 n_{m} \mu_{m}$. If the medium is extended, $H_{i}$ does not depend on whether the magnetic moments are clustered or not, as $n_{m} \sim N_{c l}^{-1}$, while $\mu_{m} \sim N_{c l}$. If $\tau_{s p}^{-1}$ is much faster than the time of nuclear moments precession $\omega_{N} \approx g_{N} \mu_{N} H_{i} / \hbar$, where $\mu_{N} \equiv e \hbar / m_{p} c$ is nuclear magneton, then the fluctuating magnetic field will induce a random walk with the step $\sim \tau_{s p}$. The change in direction of the nuclear moment per step is $\delta \theta \approx \omega_{N} \tau_{s p}$, which means that the initial phase relationship is lost after a time

$$
\tau_{n s p} \approx(1 / \delta \theta)^{2} \tau_{s p} \approx \omega_{N}^{-1} /\left(\omega_{N} \tau_{s p}\right),
$$

which should be compared with electron-nuclei decorrelation time $\tau_{n e} \approx \omega_{N}^{-1}\left(\omega_{e} / \omega_{N}\right)$ and a comparable rate of nuclei-nuclei decorrelation $\tau_{n n} \approx 0.58 \tau_{n e}\left(n_{e} / n_{n}\right)$, where $n_{e}$ and $n_{n}$ are densities of unpaired electron and nuclear spins, respectively (LD99b). Therefore, if the rate of the nuclear relaxation $\tau_{n s p}^{-1}$ can be increased in the presence of superparamagnetic inclusions by a factor $\tau_{s p} \omega_{e}$, which scales according to eq. (3) as $\exp \left(N_{c l} T_{a c t} / T\right) \omega_{e}$, provided that, nevertheless $\tau_{s p}^{-1} \gg \omega_{N}$. This means that for clusters with $N_{c l}<1.5 \times 10^{4}$ the extension of the range of frequencies for the dominant nuclear relaxation exist. If clusters are larger, then superparamagnetically enhanced nuclear relaxation gets suppresses by the factor $\left(1+\left(\omega \tau_{n s p} / 2\right)^{2}\right)^{2}$ similar to eq. (44). Figure 1 illustrates the relative roles of the Barnett and nuclear relaxation processes in paramagnetic and superparamagnetic grains and shows that for $N_{c l}=10^{4}$, nuclear relaxation get modified in the range of grain sizes smaller than $2 \times 10^{-5}$ $\mathrm{cm}$. As a result, for grains in the range from $3 \times 10^{-6}$ to $2 \times 10^{-5} \mathrm{~cm}$ superparamagnetic Barnett relaxation dominates.

The actual relaxation rate is important for getting the rates of grain flipping. The corresponding expression ac- 


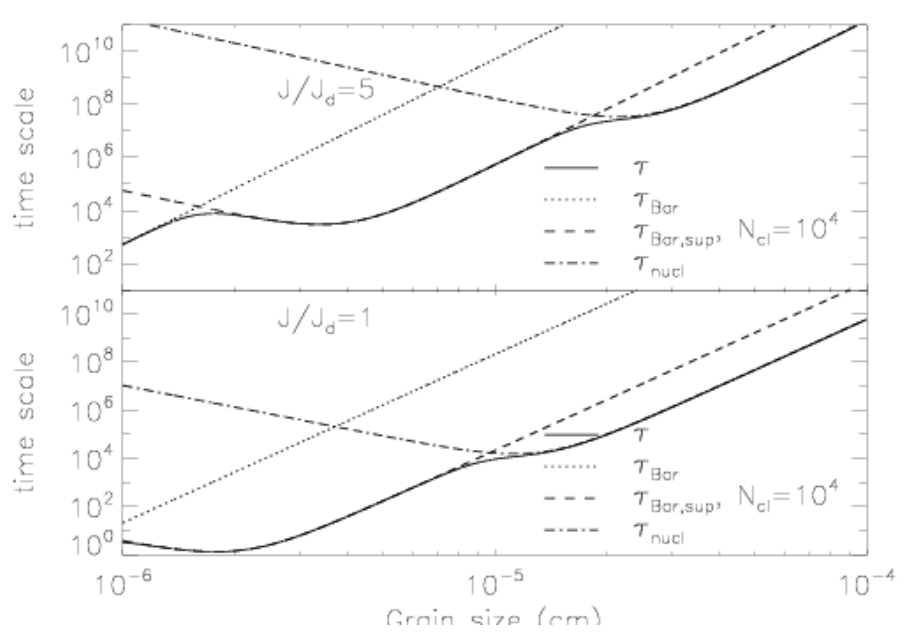

FIG. 1.- Time scales of internal relaxations: Barnett relaxation $\left(\tau_{\text {Bar }}\right)$, Barnett superparamagnetic relaxation $\left(\tau_{\text {Bar,sup }}\right)$, nuclear relaxation $\left(\tau_{n u c l}\right)$ and internal relaxation time $\left(\tau_{\text {int }}\right)$ as functions of grain size, for two values of angular momentum $J / J_{d}=5$ (upper) and $J / J_{d}=1$ (lower). Here we adopt number of iron cluster $N_{c l}=10^{4}$. Relaxation by superparamagnetic inclusion is dominant for grains smaller than $3 \times 10^{-5} \mathrm{~cm}$ for the case $J / J_{d}=1$.

cording to LD99a is $\frac{t_{t f}}{\tau_{\text {int }}}=\exp \left(J^{2} / J_{d}^{2}-0.5\right)$ where $\tau_{\text {int }}^{-1}$ is the internal relaxation rate, which is the sum of the internal relaxation rates acting on the grains, $J$ is the magnitude of the angular momentum, and $J_{d}=\sqrt{2 I_{1} k_{B} T_{d}}$ is the angular momentum of dust grain corresponding to the dust temperature $T_{d} ; I_{1}$ is the moment of the axis of major inertia $\mathbf{a}_{\mathbf{1}}$ and $k_{B}$ is the Boltzmann constant.

\section{RAT ALIGNMENT OF SUPERPARAMAGNETIC GRAINS}

As we see in Figure 2 the DG relaxation within superparamagnetic material can influence grain dynamics. The maps shown follow the evolution of an irregular grain (Shape $4^{*}$ in LD07, i.e., modified from Shape 4 by changing the helicity from left to right), keeping track of its angular momentum $J$ and the angle $\xi$ between $\mathbf{J}$ and the magnetic field. For simplicity, we disregard thermal wobbling and flipping (see Hoang \& Lazarian 2008 for more details). Comparing the panels of Figure 2, we see that without the DG relaxation (or in its presence, but assuming that grains are paramagnetic), only lower attractor point $\mathrm{B}$ is being observed in the phase trajectory map, while stationary point $\mathrm{A}$ is a repellor point (upper panel). However, this repellor point $\mathrm{A}$ becomes a new high attractor point $A^{\prime}$ when the grain is superparamagnetic (lower panel).

When gaseous bombardment is included, then, as shown in Hoang \& Lazarian (2008), grains are being kicked out from the low- $J$ attractor point and end up at the high- $J$ attractor point. Other randomization processes, e.g. shaking of a flipping grain by $\mathbf{B} \times \mathbf{v}$ electric field arising from grain drift (Weingartner 2006) or from grain emission, plasma-grain interactions etc. (see Draine \& Lazarian 1998) will have a similar effect. As a result, grain alignment with $R \approx 1$, i.e. perfect alignment of grains in respect to magnetic field, is achievable.

What are the degrees of the paramagnetic enhancement that are required to create high- $J$ attractor points? The answer depends on the angle between radiation direction and the magnetic field $\psi$ and grain properties, that, according to LH07a, can be conveniently parame-

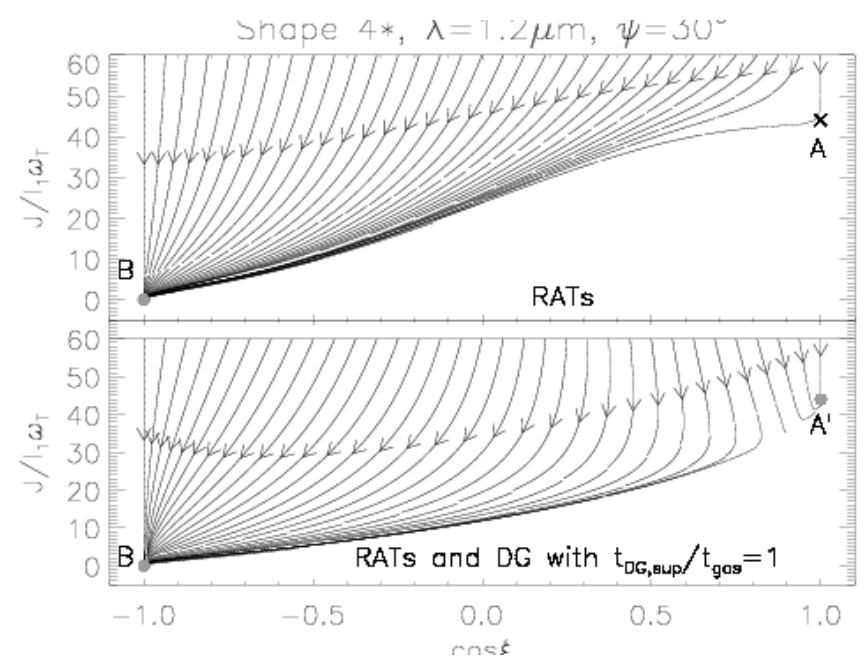

Fig. 2.- Trajectory maps for the alignment by RATs only (upper panel) and by RATs plus superparamagnetic relaxation with $t_{D G \text {,sup }} / t_{\text {gas }}=1$ (lower panel) for Shape $4^{*}$ and one light direction $\psi=30^{\circ}$. Unlike in LH07, only trajectories corresponding to $\mathbf{J}$ parallel to grain axis a are shown. Repellor points $\mathrm{A}$ in the upper panel becomes the attractor point A' in the lower panel as a result of superparamagnetic alignment.

terized by the ratio $q^{\max }=Q_{e 1}^{\max } / Q_{e 2}^{\max }$, where $Q_{e 1}^{\max }$ is the amplitude of the component of the torques parallel to vector $\mathbf{k}$ and $Q_{e 2}^{\max }$ is the amplitude of the component of the torques in the plane of grain axis of major inertia $\mathbf{a}_{1}$ and $\mathbf{k}$. Let us estimate roughly when there are high- $J$ attractor points in the presence of superparamagnetic inclusion. For simplicity, we consider the case $\psi=0^{0}$, and the default AMO with the mirror at an angle $\alpha=\pi / 4$. Thus the aligning torque reads (see eq. 25 in DW97)

$$
\begin{gathered}
\langle F(\xi)\rangle_{\phi}=\frac{M}{J^{\prime}}\left(-Q_{e 1}^{\max }\left(3 \cos ^{2} \xi-2\right) \sin \xi+Q_{e 2}^{\max } \sin 2 \xi \cos \xi\right) \\
-\frac{\sin \xi \cos \xi}{t_{D G, s p}^{\prime}},
\end{gathered}
$$

where the first term containing a factor $M=$ $\frac{u_{\text {rad }} \lambda a_{\text {eff }}^{2} t_{\text {gas }}}{2 I_{1} \omega_{T}}$, where $u_{\text {rad }}$ is the radiation energy density, $\lambda$ is the wavelength, $a_{\text {eff }}$ the effective grain size defined as the radius of the spherical grain of the same volume, $t_{g a s}$ is the gaseous damping time, denotes the aligning component of RATs (see eq. 38 in LH07) and $J^{\prime}=J / I_{1} \omega_{T}$, where $I_{1}$ is the maximal moment of inertia and $\omega_{T}$ is thermal rotational rate at $T=T_{\text {gas }}$. The second term represents the contribution from the superparamagnetic relaxation with characteristic time

$$
t_{D G, s p}^{\prime}=\frac{t_{D G, s p}}{t_{\text {gas }}} \approx 10^{2} N_{c l}^{-1} \hat{B}^{-1} \hat{K}^{-1} b_{-5}\left(\frac{\hat{n} \hat{T}_{g}^{1 / 2}}{\hat{\rho}}\right) .
$$

It is obvious from Eq. (6) that the position of the stationary points $\xi_{s}=0$ and $\pi$ (i.e. $\langle F\rangle_{\phi}=0$ ) is not affected by the presence of the additional relaxation. Also, the magnitude of spin-up torque $\langle H\rangle_{\phi}$ at this stationary point is not affected by the superparamagnetic relaxation either (see eq. 26 in DW97). As a result, the stationary point $\xi_{s}$ is an attractor point when

$$
\left.\frac{1}{\langle H\rangle_{\phi}} \frac{d\langle F\rangle_{\phi}}{d \xi}\right|_{\xi=\xi_{s}}<0 .
$$

At the stationary point $\xi_{s}=0,\langle H\rangle_{\phi}>0$, therefore, 
using Eq. (6) and (8) one gets

$$
\begin{gathered}
\frac{M}{J_{s}}\left(-Q_{e 1}^{\max }+2 Q_{e 2}^{\max }\right)-\frac{1}{t_{D G, s p}^{\prime}}<0 . \\
t_{D G, s p}^{\prime}<\frac{J_{s}}{M\left(-Q_{e 1}^{\max }+2 Q_{e 2}^{\max }\right)}=\frac{Q_{e 1}^{\max }}{\left(-Q_{e 1}^{\max }+2 Q_{e 2}^{\text {max }}\right)}(10)
\end{gathered}
$$

where we have used $J_{s} / M=\left\langle H\left(\xi_{s}=0\right)\right\rangle_{\phi}=Q_{e 1}^{\max }$ is the spin-up torque at the stationary point $\xi_{s}=0$. For $Q_{e 1}^{\max }>2 Q_{e 2}^{\max }$, the criterion (10) is always fulfilled, i.e. there is high- $J$ attractor points regardless of DG relaxation. This is similar to what obtained in LH07. When $Q_{e 1}^{\max }<2 Q_{e 2}^{\max }$, Eq. (10) gives $t_{D G \text {,sup }}^{\prime}<$ $q^{\max } /\left(2-q^{\max }\right)$ with $q^{\max }=Q_{e 1}^{\max } / Q_{e 2}^{\max }$. Thus, the AMO predicts that for $q^{\max }=0.8$, the enhancement of paramagnetic susceptibility necessary to convert the repellor points into attractor points is $t_{D G \text {, sup }}^{\prime} \leq 0.67$ for $\psi=0^{0}$. The same value $q^{\max }$ corresponds to the Shape $4^{*}$ grain at $\lambda=1.2 \mu m$ and for this grains we get the necessary value that we obtain numerically $t_{D G, \text { sup }}^{\prime} \approx 0.56$, which is close to what the AMO gives us. For experimentally studied in LH07 grain shapes the corresponding $q^{\max }$ varied from 0.4 to 10 and for $\psi$ in the range from $0^{\circ}$ to $\sim 90^{\circ}$ and found that the minimal value $t_{D G, \text { sup }}^{\prime} \approx 5 \times 10^{-2}$ is sufficient to produce the high$\mathrm{J}$ attractor points in all cases. This is easily achievable for grains having superparamagnetic inclusions with $N_{c l} \sim 5 \times 10^{3}$ iron cluster. It is clear that such superparamagnetic inclusions can substantially enhance the degree of grain alignment.

To distinguish the RAT alignment in which magnetic field is only important in terms of inducing fast Larmor precession, from a hybrid type alignment that includes an additional process, namely, superparamagnetic relaxation we shall call the latter process superamagnetic RAT alignment, or, in short, SRAT alignment. Note that superparamagnetic inclusions also enhance the rate of Larmor precession of $\mathbf{J}$ in respect to $\mathbf{B}$, which makes the alignment with respect to $\mathbf{B}$ preferable to the radiation vector $\mathbf{k}$ (see discussion in LH07a). This may allow more reliable interpretation of polarimetry in terms of magnetic field in circumstellar regions.
In terms of the RAT alignment it is possible to show that even inclusions with $N \sim 10^{3}$ can result in the appearance of a new high- $J$ attractor point. As a result, the RAT alignment can be enhanced substantially, becoming nearly perfect. This alignment is different from the alignment described in Draine \& Weingartner (1996), however. In that paper, it was assumed that RATs provide the spin-up similar to pinwheel torques in Purcell (1979), while ordinary paramagnetic dissipation does the job of alignment. The latter interpretation of the RAT action is erroneous, as RATs provide the alignment of their own, making the weak paramagnetic torques irrelevant. It is only after being enhanced through the presence of superparamagnetic inclusions, that the paramagnetic dissipation can affect the alignment. If observations prove that perfect alignment of grains is required, $S R A T$ alignment can achieve this. Interestingly enough, the synthesis of pinwheel torques and superparamagnetic inclusions proposed in Spitzer \& McGlynn (1979), does not work for interstellar medium, as superparamagnetic grains are strongly thermally trapped and cannot rotate suprathermally, unless RATs are present. Note, that superparamagnetic inclusions will also enhance the mechanical alignment of helical grains. Such an alignment was proposed in Lazarian \& Hoang (2007b) where it was shown that torques arising from the gas-grain drift may act on irregular grains the same way as RATs do.

Our results above can be briefly summarized as follows:

- Superparamagnetic inclusions affect both Barnett and nuclear relaxation, but in a different way: Barnett relaxation is being enhanced by the increase of magnetic susceptibility, while the nuclear relaxation does not changes its amplitude, getting, nevertheless extended for higher frequencies.

- Supraparamagnetic inclusions modify the RAT alignment and RAT-superparamagnetic mechanism, which we termed SRAT alignment, may provide higher degree of alignment compared to the RAT mechanism alone.

- The results above are also applicable to mechanical alignment of irregular grains.

Acknowledgments The work was supported by the NSF Center for Magnetic Self-Organization in Laboratory and Astrophysical Plasmas and NSF grant AST 0507164 .

\section{DISCUSSION AND SUMMARY}

\section{REFERENCES}

Aiken D. K., Efstathiou A., McCall A., Hough J. H. 2002, MNRAS, 329, 647

Andersson B-G, \& Potter S.B. 2007, ApJ, 665, 369

Barnett, S.J. 1915, Phys. Rev., 6, 239

Bethell T., Cherpunov A., Lazarian A., Kim J. 2007, ApJ, 663, 1055

Billas, I.M., Chatelain, A., \& de Heer, W.A. 1994, Science, 265, 1682

Cho J., Lazarian A. 2005, ApJ, 631, 361 (CL05)

Cho J.,\& Lazarian A. 2007, ApJ, 669, 1085

Dolginov A.Z., Mytrophanov I.G. 1976, Ap\&SS, 43, 291

Djouadi, Z., Gattacceca, J., D'Hendecourt, L., Rochette, P., Jones, A. P., Davoisne, C., Leroux, H., \& Borg, J. 2007, A\& A, 468,9

Draine, B. 1996, in Polarimetry of the Interstellar Medium, eds W R Roberge and D. C. B. Whittet, APS series, Volume 1997, p. 16

Draine B., Flatau P. 1994, J. Opt. Soc. Am. A., 11, 1491
Draine, B., Lazarian, A. 1999, ApJ, 512, 740

Draine, B., Weingartner, J. 1996, ApJ, 470, 551 (DW96)

Draine, B. Weingartner, J. 1997, ApJ, 480, 633 (DW97)

Goodman, A. \& Whittet, D. C. B. 1995, ApJ, 455, 181

Hall J. 1949, Science, 109, 166

Harwit M. 1970, Nature, 226, 61

Hoang T, Lazarian A. 2008, MNRAS, in press

Kneller, E.F. \& Laborsky, F.E. 1963, J. Appl. Phys. 34, 656

Lazarian A. 2007, J. Quant. Spectrosc. Rad. Trans., 106, 225

Lazarian A. 1994, MNRAS, 268, 713

Lazarian A. 1995, ApJ, 453, 229

Lazarian A. \& Draine B. 1999a, ApJ, 516, L37 (LD99a)

Lazarian A. \& Draine B. 1999b, ApJ, 520, L67 (LD99b)

Lazarian, A. \& Efroimsky, M. 1998

Lazarian, A. \& Hoang, T. 2007a, MNRAS (LH07)

Lazarian A., Hoang T. 2007b, ApJL, 669, L77

Lazarian A., Roberge W. 1997, ApJ, 484, 230

Martin, P. 1995, ApJ, 445, 63 
Morrish, A.H. 1980, The Physical Principles of Magnetism, Huntingdon: Krieger

Rosenbush V., Kolokolova L.,Lazarian A., Shakhovskoy N., Kiselev N. 2007, Icarus, 186, 317

Spitzer L., McGlynn T. 1979, ApJ, 231, 417

Weingartner J., \& Draine B. 2003, ApJ, 589, 283
Weingartner J. 2006, ApJ, 647, 390

Whittet D.C.B, Hough J.H, Lazarian A., Hoang T. 2008, ApJ, $674,000-000$

van Vleck, J.H. 1937, J. Chem. Phys., 5, 320 